\title{
The History and Properties of Ultrasonic Inhomogeneous Waves
}

\author{
Nico F. Declercq, Member, IEEE, Rudy Briers, Joris Degrieck, and Oswald Leroy, Senior Member, IEEE
}

\begin{abstract}
This paper gives a historical survey of the development of the inhomogeneous wave theory, and its applications, in the field of ultrasonics. The references are listed predominantly chronologically and are as good as complete. Along the historical description, several scientific features of inhomogeneous waves are described. All topics of inhomogeneous wave research are taken into account, such as waves in viscoelastic solids and liquids, thermoviscous liquids and solids, and anisotropic viscoelastic materials. Also inhomogeneous waves having complex frequency are described. Furthermore, the formation of bounded beams by means of inhomogeneous waves is given and the diffraction of inhomogeneous waves on periodically corrugated surfaces. The experimental generation of inhomogeneous waves is considered as well.
\end{abstract}

\section{INTRODUCTION}

$\mathrm{T}$ HERE is no better sentence to start this paper than one which is similar to the one that was used to end a writing by Poirée [44] (i.e., 'Ultrasonic inhomogeneous waves are a treasure"). It will be seen in what follows that inhomogeneous waves have been a gold mine for many researchers because it formed the core of their scientific activities for many years. But it also will be seen that inhomogeneous waves are actually a treasure of physics because of many theoretically predictable, beautiful features, properties, and behaviors. Furthermore, it will be noticed that inhomogeneous waves often let nature poke fun at humans' intuition.

We would like to show first that inhomogeneous waves are of primordial importance in the correct description of reflection/transmission phenomena. For example, take a look at the interface between a viscoelastic liquid and an ideal liquid. A homogeneous plane wave incident from the viscoelastic fluid is damped along its propagation direction. The transmitted wave is not damped along its propagation direction, but its amplitude is influenced by the one of the incident wave as shown in Fig. 1. It can be noticed in Fig. 1 that the transmitted wave will have an amplitude that grows exponentially along its wave front.

Manuscript received December 1, 2003; accepted June 9, 2004. Work supported by The Flemish Institute for the Encouragement of the Scientific and Technological Research in Industry (I.W.T.).

N. F. Declercq and J. Degrieck are with the Soete Laboratory, Department of Mechanical Construction and Production, Ghent University, B-9000 Ghent, Belgium (e-mail: NicoF.Declercq@UGent.be).

R. Briers is with Katholieke Hogeschool West Vlaanderen (departement regentaat en normaalschool), Sint Jozefstraat 1, 8820 Torhout, Belgium.

O. Leroy is with the Interdisciplinary Research Center, Katholieke Universiteit Leuven Campus Kortrijk, B-8500 Kortrijk, Belgium.

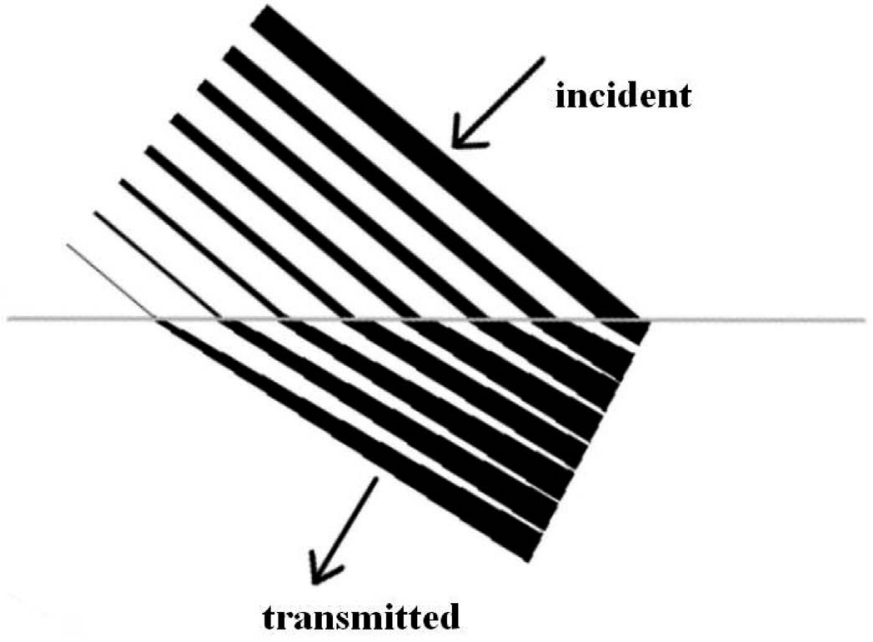

Fig. 1. An inhomogeneous wave is naturally "born" in transmission at the interface between a viscoelastic liquid (top) and an ideal liquid (bottom). In general, whenever damping plays a role, inhomogeneous waves are part of the game. The black lines correspond to wave fronts; their thickness corresponds to the amplitude.

Such waves are called inhomogeneous waves. Therefore, inhomogeneous waves are entities that are necessary to describe even such a simple phenomenon as scattering on plane interfaces.

For some people, inhomogeneous waves are completely unknown, for others they are unavoidable when the interaction of sound with viscoelastic materials is to be described. Yet other people (the inhomogeneous wave enthusiasts) study such waves in the bulk of a material (for example, generated by means of some sort of a transducer), and let them interact with materials; such waves are often explicitly called inhomogeneous bulk waves. Because inhomogeneous waves are the most general plane wave solutions of the wave equation, there is actually no reason why one should neglect inhomogeneous waves and only study the very trivial case of homogeneous plane waves.

This paper describes from a historical point of view how the concept of inhomogeneous waves in ultrasonics has grown from an artifact to a fortune of physics. The references in this paper are listed predominantly according to their submission date, and the authors have tried to make this list complete. The historical approach is the 'red line' in this paper, and the scientific knowledge is distributed along the paper as to appear whenever necessary to understand the historical development. Inhomogeneous waves have become tools for nondestructive testing. There- 


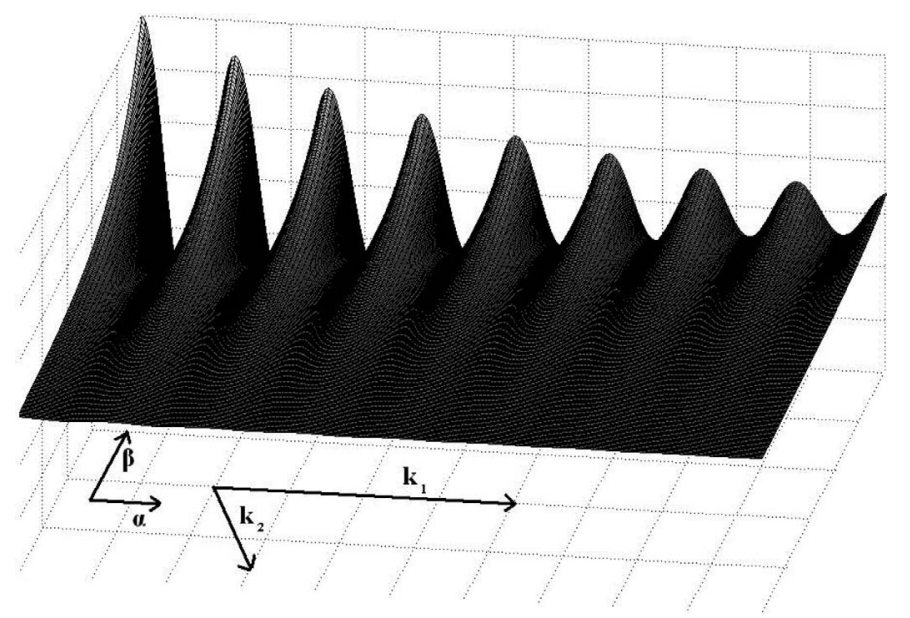

Fig. 2. An inhomogeneous wave. Damping occurs along the propagation direction and there is also an exponential amplitude decay along the wave front due to the inhomogeneity.

fore, it is expected that many applications will appear in papers in the coming decade. The authors would like to express their wish that this paper may become an inspiring source for future scientists in this fantastic field of research.

In the past, several papers have appeared that give an overview of inhomogeneous waves, but never in a historical context and never spanning the whole area of inhomogeneous waves. In 1988, an overview appeared by Poirée [31], dealing with elastic solids and ideal fluids and one by Deschamps and Changlin [32], dealing with viscoelastic materials, conservation of energy, and special attention also went to the generalized law of Snell-Descartes. A year later came another review by Leroy et al. [33]. Later reviews can be found in Deschamps [39] (mainly dealing with thermoviscous materials and viscoelastic isotropic and anisotropic materials) and Poirée [44]. Leroy also wrote a review in 1996 [67] with special attention for nonspecular bounded beam effects at the Rayleigh angle of incidence. The current paper deals with all important aspects of inhomogeneous waves and does not go into detail in some areas that have been less important for the growth of the field of inhomogeneous waves in ultrasonics. These areas are dealing with waves in sediment layers [43], in inhomogeneous media such as rock layers [59], viscous fluids [63], porous materials [24], [71] and the interaction of inhomogeneous waves with defects [83]. However, it is believed that the latter area will become more and more important in the coming decade as inhomogeneous waves are starting to be used in nondestructive testing (NDT).

\section{Basic Properties of Ultrasonic INHOMOGENEOUS WAVES}

An example of an inhomogeneous wave is shown in Fig. 2. The different parameters denoted in Fig. 2 will be outlined below. This paragraph summarizes some basic properties of inhomogeneous waves. How these properties were discovered and supplementary developments will be described in subsequent paragraphs.

Basically an inhomogeneous ultrasonic wave, represented by a particle displacement $\mathbf{u}$, is written just as a homogeneous plane wave, i.e.:

$$
\mathbf{u}=A \mathbf{P} \exp (i \mathbf{k} \bullet \mathbf{r}-i \omega t)
$$

where $A$ is the amplitude, $\mathbf{P}$ is the polarization, $\mathbf{k}$ is the wave vector, $\omega$ is the angular frequency, $\mathbf{r}$ is the position, $t$ is time, and $i=\sqrt{-1}$. tion:

The wave (1) is a solution of the viscoelastic wave equa-

$$
\tilde{\mu} \Delta \mathbf{u}+(\tilde{\lambda}+\tilde{\mu}) \nabla \nabla \cdot \mathbf{u}=\rho \ddot{\mathbf{u}}
$$

with

$$
\begin{aligned}
& \tilde{\lambda}=\lambda+\lambda^{\prime} \frac{\partial}{\partial t} \\
& \tilde{\mu}=\mu+\mu^{\prime} \frac{\partial}{\partial t}
\end{aligned}
$$

if

$$
\mathbf{k} \cdot \mathbf{k}=\rho \omega^{2} /(\tilde{\lambda}+2 \tilde{\mu})
$$

and

$$
\mathbf{k} \times \mathbf{P}=0 .
$$

or if

$$
\mathbf{k} \cdot \mathbf{k}=\rho \omega^{2} / \tilde{\mu}
$$

and

$$
\mathbf{k} \cdot \mathbf{P}=0
$$

Waves that correspond to (4) and (5) are called longitudinal, whereas waves corresponding to (6) and (7) are called shear waves.

It also is convenient to take into account that $\partial / \partial t \approx$ $-i \omega$ and use the following substitutions in (4) and (6):

$$
\begin{gathered}
\mu=\rho \omega^{2} \frac{v_{s}^{2}\left(\omega^{2}-\alpha_{0, s}^{2} v_{s}^{2}\right)}{\omega^{4}+2 \omega^{2} \alpha_{0, s}^{2} v_{s}^{2}+\alpha_{0, s}^{4} v_{s}^{4}}, \\
\mu^{\prime}=-2 \rho \omega^{2} v_{s}^{3} \frac{\alpha_{0, s}}{\omega^{4}+2 \omega^{2} \alpha_{0, s}^{2} v_{s}^{2}+\alpha_{0, s}^{4} v_{s}^{4}}, \\
\lambda=\frac{-\rho \omega^{2} M}{S}, \\
\lambda^{\prime}=\frac{2 \rho \omega^{2} N}{S},
\end{gathered}
$$

with 


$$
\begin{aligned}
& M=\omega^{6}\left(2 v_{s}^{2}-v_{d}^{2}\right) \\
& +\omega^{4}\left(\alpha_{0, d}^{2} v_{d}^{4}-2 v_{d}^{2} \alpha_{0, s}^{2} v_{s}^{2}+4 v_{s}^{2} \alpha_{0, d}^{2} v_{d}^{2}-2 v_{s}^{4} \alpha_{0, s}^{2}\right) \\
& +\omega^{2}\left(-v_{d}^{2} \alpha_{0, s}^{4} v_{s}^{4}-4 v_{s}^{4} \alpha_{0, d}^{2} v_{d}^{2} \alpha_{0, s}^{2}+2 \alpha_{0, d}^{2} v_{d}^{4} \alpha_{0, s}^{2} v_{s}^{2}\right. \\
& \left.+2 v_{s}^{2} \alpha_{0, d}^{4} v_{d}^{4}\right)+\alpha_{0, d}^{2} v_{d}^{4} \alpha_{0, s}^{4} v_{s}^{4}-2 v_{s}^{4} \alpha_{0, d}^{4} v_{d}^{4} \alpha_{0, s}^{2}, \\
& N=2 \alpha_{0, d}^{4} v_{d}^{4} v_{s}^{3} \alpha_{0, s}+4 \omega^{2} v_{s}^{3} \alpha_{0, d}^{2} v_{d}^{2} \alpha_{0, s}-2 \omega^{2} v_{d}^{3} \alpha_{0, s}^{2} v_{s}^{2} \alpha_{0, d} \\
& -v_{d}^{3} \alpha_{0, s}^{4} v_{s}^{4} \alpha_{0, d}+2 \omega^{4} v_{s}^{3} \alpha_{0, s}-\omega^{4} v_{d}^{3} \alpha_{0, d}, \\
& S=\left(\omega^{4}+2 \omega^{2} \alpha_{0, s}^{2} v_{s}^{2}+\alpha_{0, s}^{4} v_{s}^{4}\right)\left(\omega^{4}+2 \alpha_{0, d}^{2} v_{d}^{2} \omega^{2}+\alpha_{0, d}^{4} v_{d}^{4}\right),
\end{aligned}
$$

then (4) becomes:

$$
\mathbf{k} \cdot \mathbf{k}=\left(\frac{\omega}{v_{d}}-i \alpha_{0, d}\right)^{2}
$$

and (6) becomes:

$$
\mathbf{k} \cdot \mathbf{k}\left(\frac{\omega}{v_{s}}-i \alpha_{0, s}\right)^{2}
$$

In (15) and (16) $v_{d}$ and $v_{s}$ are the longitudinal, respectively, shear wave velocity for pure homogeneous plane waves in the considered medium, and $\alpha_{0, d}$ and $\alpha_{0, s}$ are the intrinsic damping coefficients in the medium for longitudinal, respectively, shear waves.

Whenever $\mu^{\prime}$ and $\lambda^{\prime}$ are nil, it is perfectly possible that all quantities in (1) are real valued. The amplitude $A$ can be real or complex, depending on the phase at the origin. Whenever $\mu^{\prime}$ and/or $\lambda^{\prime}$ differ from zero, it follows from (4) and (6) that $\mathbf{k}$ is complex valued. In addition, regardless of the values of $\mu^{\prime}$ and $\lambda^{\prime}, \mathbf{k}$ can be complex valued because of specific boundary conditions. Hence, inhomogeneous waves are defined as waves described by (1) and having a complex wave vector $\mathbf{k}$, i.e.:

$$
\mathbf{k}=\mathbf{k}_{1}+i \mathbf{k}_{2} \text {. }
$$

A summation of a real vector with an imaginary vector, as in (17), is called the bivector formalism. Substitution of (17) in (1) reveals that $\mathbf{k}_{1}$ influences the phase of the considered sound wave, and $i \mathbf{k}_{2}$ influences the amplitude. Therefore, it is common to write:

$$
\mathbf{k}_{2}=\boldsymbol{\alpha}-\boldsymbol{\beta},
$$

with

$$
\boldsymbol{\alpha} \| \mathbf{k}_{1},
$$

and

$$
\boldsymbol{\beta} \perp \mathbf{k}_{1},
$$

$\boldsymbol{\alpha}$ is called the damping vector, and $\boldsymbol{\beta}$ is called the inhomogeneity vector.

Now if $\mathbf{k}$ is indeed complex, then from (5) and (7), it follows that the polarization vector $\mathbf{P}$ also can be complex, i.e.:

$$
\mathbf{P}=\mathbf{P}_{1}+i \mathbf{P}_{2}
$$

The vector $\mathbf{P}$ is normalized when:

$$
\mathbf{P} \cdot \mathbf{P}=1 \rightarrow\left\{\begin{array}{l}
\mathbf{P}_{1} \cdot \mathbf{P}_{1}=1+\mathbf{P}_{2} \cdot \mathbf{P}_{2} \\
\mathbf{P}_{1} \cdot \mathbf{P}_{2}=0
\end{array} .\right.
$$

An important feature of the polarization is consequently that the real component is always larger than the imaginary component.

Because of (21), the values of $\mathbf{P}_{1}$ and $\mathbf{P}_{2}$ are determined by (5) or (7) as follows:

For longitudinal waves:

$$
\mathbf{k} \times \mathbf{P}=0 \rightarrow \mathbf{P}=\mathbf{k} /|\mathbf{k}| \rightarrow \mathbf{P}=\mathbf{k}\left(\omega / v_{d}-i \alpha_{0, d}\right)^{-1 / 2}
$$

or, in other words:

$$
\begin{aligned}
& \mathbf{P}_{1}=\frac{\frac{\omega}{v_{d}} \mathbf{k}_{1}-\alpha_{0, d} \mathbf{k}_{2}}{\frac{\omega^{2}}{v_{d}^{2}}+\alpha_{0, d}^{2}}, \\
& \mathbf{P}_{2}=\frac{\alpha_{0, d} \mathbf{k}_{1}+\frac{\omega}{v_{d}} \mathbf{k}_{2}}{\frac{\omega^{2}}{v_{d}^{2}}+\alpha_{0, d}^{2}},
\end{aligned}
$$

while for shear waves:

$$
\begin{aligned}
\mathbf{k} \cdot \mathbf{P}=0 \rightarrow \mathbf{P}=\mathbf{F} \times \mathbf{k} /|\mathbf{k}| \rightarrow \mathbf{P} \\
=(\mathbf{F} \times \mathbf{k})\left(\omega / v_{s}-i \alpha_{0, s}\right)^{-1 / 2},
\end{aligned}
$$

or, in other words:

$$
\begin{aligned}
& \mathbf{P}_{1}= \frac{\frac{\omega}{v_{s}} \mathbf{F} \times \mathbf{k}_{1}-\alpha_{0, s} \mathbf{F} \times \mathbf{k}_{2}}{\frac{\omega^{2}}{v_{s}^{2}}+\alpha_{0, s}^{2}}, \\
& \mathbf{P}_{2}=\frac{\alpha_{0, s} \mathbf{F} \times \mathbf{k}_{1}+\frac{\omega}{v_{s}} \mathbf{F} \times \mathbf{k}_{2}}{\frac{\omega^{2}}{v_{s}^{2}}+\alpha_{0, s}^{2}},
\end{aligned}
$$

with $\mathbf{F} \perp \mathbf{k}$.

From (24) we learn that longitudinal inhomogeneous waves are elliptically polarized with the ellipse of particle motion inside the plane formed by the real and imaginary part of the wave vector. From (26) we learn that shear waves are also elliptically polarized. However, the ellipse of particle motion no longer is inside the plane formed by the real and imaginary part of the wave vector. These polarization properties involve that it would be more appropriate to talk about quasilongitudinal and quasishear waves just as in anisotropic media, but this is seldom done in inhomogeneous wave theory.

In isotropic materials, it is very convenient to let relations (4)-(7) automatically be taken into account by working with the Helmholtz decomposition:

$$
\mathbf{u}=\nabla \varphi+\nabla \times \psi \mathbf{e}
$$


where $\varphi$ and $\psi$ are scalar plane waves (having a complex wave vector) and $\mathbf{e}$ is an appropriately chosen unit vector so that:

$$
\nabla \cdot \psi \mathbf{e}=0
$$

In anisotropic materials, it is better to work directly with (1).

In many papers, the dispersion relations (15) or (16) are written in terms of the components of $\mathbf{k}$, leading, for $m=s$ or $m=d$, to:

$$
k_{1}^{2}-\beta^{2}-\alpha^{2}=\frac{\omega^{2}}{v_{m}^{2}}-\alpha_{0, m}^{2},
$$

and

$$
k_{1} \alpha=\frac{\omega}{v_{m}} \alpha_{0, m} .
$$

For the moment we have supposed that (1) is a harmonic function of time. Implicitly this means that we supposed that the vibration is somehow generated by means of a harmonic source. However, there also are other sources, such as damped or even critically damped sources, possible. Sound coming from such sources will have different characteristics in time and space. In fact, the source affects the complex amplitude but also the frequency when, depending on the characteristics of the source, this frequency can be real (harmonic), complex (harmonically damped), or even imaginary (critically damped). Hence, in general:

$$
\omega=\omega_{1}+i \omega_{2}
$$

The concept of complex frequencies will be dealt with in detail later.

\section{The Concept of Complex Angles}

In the early days of the inhomogeneous wave theory and in mathematical papers about this theory, the concept of complex angles is often used.

From the fact that [see (15) and (16)], for $m=s$ or $m=d$ :

$$
\mathbf{k} \cdot \mathbf{k}=\left(\frac{\omega}{v_{m}}-i \alpha_{0, m}\right)
$$

and

$$
\sin ^{2} \theta+\cos ^{2} \theta=1,
$$

in the XZ-plane $\mathbf{k}$ can be formally written as:

$$
\mathbf{k}=\left(\frac{\omega}{v_{m}}-i \alpha_{0, m}\right)\left(\mathbf{e}_{x} \sin \theta+\mathbf{e}_{z} \cos \theta\right) .
$$

Identification with:

$$
\mathbf{k}=\left(k_{1, x}+i k_{2, x}\right) \mathbf{e}_{x}+\left(k_{1, z}+i k_{2, z}\right) \mathbf{e}_{z},
$$

then results in

$$
\begin{aligned}
& k_{1, x}=\frac{\omega}{v_{m}} \sin \theta_{1} \cosh \theta_{2}+\alpha_{0, m} \cos \theta_{1} \sinh \theta_{2}, \\
& k_{2, x}=\frac{\omega}{v_{m}} \cos \theta_{1} \sinh \theta_{2}-\alpha_{0, m} \sin \theta_{1} \cosh \theta_{2}, \\
& k_{1, z}=\frac{\omega}{v_{m}} \cos \theta_{1} \cosh \theta_{2}-\alpha_{0, m} \sin \theta_{1} \sinh \theta_{2}, \\
& k_{2, z}=-\frac{\omega}{v_{m}} \sin \theta_{1} \sinh \theta_{2}-\alpha_{0, m} \cos \theta_{1} \cosh \theta_{2},
\end{aligned}
$$

with

$$
\theta=\theta_{1}+i \theta_{2}
$$

Hence the angle defined in (34) is a complex angle.

\section{The Generalized Law of Snell-Descartes}

When an inhomogeneous wave is incident on an interface between two media, reflected as well as transmitted inhomogeneous waves will be generated, at least if the mechanical continuity conditions allow them to be generated. The conditions are very often given by continuity of normal displacement and normal stress-vector for liquid-solid interfaces and total stress for solid-solid interfaces. However, there is also the continuity of propagation-phase, which simply states that along the interface (for example, parallel with the $\mathrm{X}$-axis) incident sound coincidentally generates scattered sound. This principle is called the classical law of Snell-Descartes and is physically best formulated as continuity of:

$$
\mathbf{k}_{1} \cdot \mathbf{r} \text { for } \mathbf{r} \in \text { interface, }
$$

and

$$
\text { continuity of } \omega_{1} \text {. }
$$

In linear acoustics, however, there is also the principle that the amplitude of the scattered sound is linear dependent on the incident amplitude. If the incident amplitude differs from spot to spot (and/or from time to time) along the interface because this incident wave is inhomogeneous (and/or has complex frequency), then the scattered waves must be equally profiled in space (and/or in time) along the interface. This results in continuity of:

$$
\mathbf{k}_{2} \cdot \mathbf{r} \text { for } \mathbf{r} \in \text { interface, }
$$

and

$$
\text { continuity of } \omega_{2} \text {. }
$$

Eq. (38)-(41) can be compressed as continuity of:

$$
\mathbf{k} \cdot \mathbf{r} \text { for } \mathbf{r} \in \text { interface, }
$$

and

$$
\text { continuity of } \omega \text {. }
$$


The principles formulated in (42) and (43) are called the generalized law of Snell-Descartes. The principle is schematically shown in Fig. 3, for an interface between two liquids. The projections of each wave vector on the interface are the same for each of the present waves (incident 'inc', reflected ' $r$ ' and transmitted ' $t$ ').

\section{The Founder of the Ultrasonic Inhomogeneous WaVe Theory}

In numerous papers concerning inhomogeneous waves, it can be found that Brekhovskikh [1] already mentions the fact that the wave number can be complex. The pioneering work of Cooper and Reiss [2] and Cooper [3] is almost never mentioned. Most likely this work was forgotten by the refounders of ultrasonic inhomogeneous waves more than a decade later. However the work of Cooper [3] can be considered as the foundation of the ultrasonic inhomogeneous wave theory. In this work, the existence of inhomogeneous waves (Cooper named them general plane waves) in viscoelastic media is proved, and the scattering of such waves on an isotropic solid-vacuum interface and an interface between two isotropic solids is considered. Note that there are some printing errors in Cooper's paper [3] that were discovered only in 1987 by Jones et al. [27]. Even less known is the fact that Cooper already implements what has later been called the generalized law of Snell-Descartes, see (42) and (43), and the dispersion relation (32) for inhomogeneous waves. Cooper does not apply any bivector formalism and does not consider the dispersion relation in its modern form, resulting from that bivector formalism. Cooper also did not discover the fact that a reflection coefficient may exceed unity. Nevertheless, there can be no doubt at all that Cooper [3] should be considered the real founder of ultrasonic inhomogeneous wave theory.

\section{The Refounders and Boosters of the Ultrasonic Inhomogeneous Wave Theory}

\section{A. Single Ultrasonic Inhomogeneous Waves}

In his paper, Frisk [6] deals with the fact that the description of the radiation field of tiny sources cannot occur without involving inhomogeneous waves. Therefore, he studies the reflection of such waves from interfaces in the ocean bottom and states that Snell's law for real wave vector components can be generalized to the case of complex wave vector components without any problem. He also describes the fact that the reflection coefficient can exceed unity without violation of the principle of energy conservation. However, Frisk studies inhomogeneous waves not as real physical entities, but as parts of the decomposition of the sound field produced by tiny sources. Therefore, his inhomogeneous waves are of such a kind that they damp away from the source and would nowadays be called

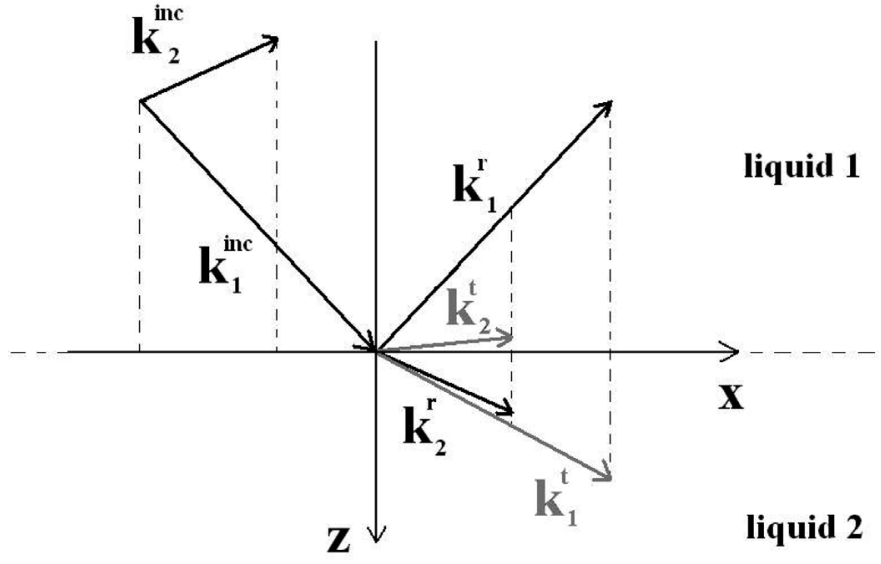

Fig. 3. Schematic of what is called the generalized law of SnellDescartes. All real wave vectors involved have the same projection on the interface and the same holds for the imaginary wave vectors.

damped homogeneous plane waves. As a result, inhomogeneous waves in Frisk's paper are only important very close to the sound source.

Because the work of Cooper and Reiss [2] and Cooper [3] seemed to be forgotten, in the period of Frisk [6], only those researchers were interested in inhomogeneous waves who also were interested in near-field phenomena.

New life was given to the theory by an error in a paper of Atalar [7]. Atalar dealt with a lossy liquid-lossless isotropic solid interface and he explicitly prevented exponential amplitude growth away from the interface. The reason originated from the then well established idea (the Sommerfeld radiation condition) that the amplitude always must be zero at infinity. However Sommerfeld's rule is not really crucial if one is considering inhomogeneous incident waves. Apparently Atalar was not aware of this. The paper of Atalar shows a reflection coefficient that is so extraordinary that it inspired Claeys and Leroy [11] to study it deeper. They came to the conclusion that, contrary to what Atalar had proposed, the transmitted waves in the case studied by Atalar always must be directed in such a way that there is exponential growth inside the solid. In a much later study, Deschamps and Roux [37] and Roux [45] have studied this problem in more detail; and they came to the conclusion that the classical Sommerfeld radiation condition should not be used for inhomogeneous waves, stating that, for surface waves, it is not always necessary to impose that their amplitude should tend to zero at large distances away from the interface. Atalar [12] resolved this discussion by stating that Claeys and Leroy [11] were right below the critical angle, and that he was right beyond the critical angle. Hence, the discussion of Atalar [12] and Claeys and Leroy [11] resulted in what would later be discovered experimentally by Deschamps [61]. These discussions also showed that ultrasonic inhomogeneous waves were concepts that were naturally generated during scattering whenever damping becomes a factor of importance. 


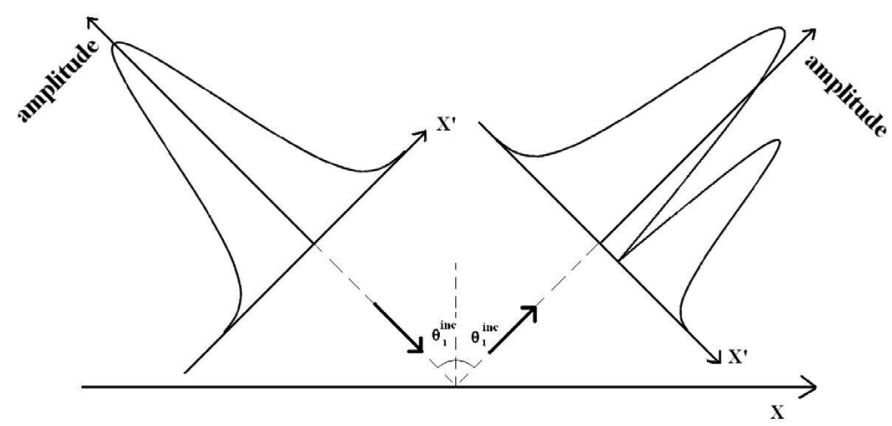

Fig. 4. A bounded beam with profile $f\left(x^{\prime}\right)$ is incident on the left and is reflected on the right.

One month later than Claeys and Leroy submission [11], Weston [10] published a paper that also dealt with inhomogeneous waves. Weston was interested in very small inhomogeneities of sound in waveguides [8]. For such small inhomogeneities, there are regions in the angular interval $\left[0, \frac{\pi}{2}\right]$ in which the reflection coefficient has practically constant amplitude. This lead Weston to consider a formulation, like Brekhovskikh [1] and Bertoni and Tamir [98] did in the framework of homogeneous plane waves, of a beam shift due to constant amplitude and high-phase shifts in a beam that is composed (in Fourier sense) of very weakly inhomogeneous waves. Even though Weston's considerations practically only hold for guided waves in between lossy media, an important conclusion of his paper is that there can be an energy gain when inhomogeneous waves are reflected. Even more interesting is a crucial sentence in Weston's paper [8] that would become one of the major ideas of inhomogeneous wave theorists, i.e., "In fact, there are the two viewpoints on the reflection of inhomogeneous waves, and it is worth stressing that these are equivalent: a displacement like other waves with no extra gain, or no displacement but with gain." This intuition was shared by Leroy et al. and they later proved [26] explicitly that there is energy conservation when inhomogeneous waves are reflected from an interface between two media. This intuition also formed the basic idea in unraveling the Schoch displacement [13].

\section{B. The Formation of Bounded Beams by Means of Inhomogeneous Waves and Their Connection with Leaky Surface Waves}

The real breakthrough of inhomogeneous waves originated from work by Claeys and Leroy [13] and Claeys [18] in which they described a bounded beam as a superposition of inhomogeneous waves by means of the Prony technique. Hence, their superposition is performed not in Fourier-sense but in a sense that had only been done before in electronics and only in the time domain, e.g., Spitznogle and Quazi [4].

Essentially the method works as follows: In Fig. 4, a schematic is given of the different coordinates that are used below. On the left there is the incident (Gaussian) bounded beam with given profile; on the right there is the reflected profile. The bounded beam $f(x, y)$ with profile $f\left(x^{\prime}\right)$ is written as:

$$
f\left(x^{\prime}\right)=\sum_{n=-N}^{N} I_{n} A_{n} \exp \left(\beta_{n} x^{\prime}\right)
$$

with $I_{n}=0$ if $n<0$ and $I_{n}=1$ if $n \geq 0$ in the original work of Claeys and Leroy [13] and $I_{n} \equiv 1$ in a later extension of the original method (see further below).

For symmetrical profiles this leads to:

$$
f\left(x^{\prime}\right)=A_{0}+\sum_{n=1}^{N}\left(I_{n}+I_{-n}\right) A_{n} \exp \left(\beta_{n} x^{\prime}\right) .
$$

The transformation $\beta_{n}=n / p$ and $x^{\prime}=p \ln \gamma$ consequently results in:

$$
A_{0}+\sum_{n=1}^{N}\left(I_{n}+I_{-n}\right) A_{n} \gamma^{n}=f(p \ln \gamma) .
$$

The right side of (46) also can be decomposed in Laguerre polynomials $L_{n}$ of order $n$ as:

$$
f(p \ln \gamma)=B_{0}+\sum_{n=1}^{N} B_{n} L_{n}(\gamma)
$$

with

$$
B_{n}=\int_{0}^{\infty} \exp (-\gamma) f(p \ln \gamma) L_{n}(\gamma) d \gamma .
$$

The unknown coefficients $A_{n}$ then are found elegantly by means of linear combinations of $B_{n}$ or directly just by means of a numerical optimization of (45). This superposition lead to simulations of the interaction of bounded Gaussian beams at all angles of incidence. Especially the simulation of the Schoch effect at the Rayleigh angle and at the Lamb angle, which had up until then only been done by means of the Fourier method for all angles of incidence and a more specialized form of the Fourier method at the Rayleigh angle [98], awakened many acousticians. From that moment on there existed an undisputable bond between inhomogeneous waves and leaky surface waves. Now, since leaky surface waves were known to be of extraordinary importance in nondestructive testing, the breakthrough of the ultrasonic inhomogeneous wave theory was a fact.

In later papers, Leroy et al. [21], Leroy et al. [26], and Sebbag [28] highlighted the difference in the reflection coefficient at the Rayleigh angle of homogeneous plane waves and inhomogeneous plane waves. Inhomogeneous plane waves, as used in the bounded beam decomposition of Claeys and Leroy [13], and Leroy [68], showed a minimum at the Rayleigh angle that was in intuitive agreement with energy transformation from incident sound to Rayleigh waves, but nothing like this was visible for homogeneous plane waves. This together with their experience 
in the field of acousto-optics, lead to a new definition of the reflection coefficient for bounded beams. This coefficient was the complex amplitude of the reflected sound integrated over the whole profile, divided by the one of the incident beam. This coefficient showed a minimum at the Rayleigh angle, in agreement with intuition. Furthermore, their definition was in accordance with a measurable entity in acousto-optics, in which the second order diffracted light amplitude corresponded to their definition of the reflection coefficient and was directly measurable.

A vast study that revealed the fact that inhomogeneous waves are excellent tools to excite leaky Rayleigh waves was performed by Poirée and Sebbag [36] and Quentin et al. [38]. They showed not only that leaky Rayleigh waves could be written as a combination of inhomogeneous waves, but also that it was from a physical point of view natural to generate such waves by means of incident inhomogeneous waves. Later, Duclos et al. [52] also performed a study on the connection of Lamb waves with inhomogeneous waves.

Several years after the findings of Claeys and Leroy [13], Van den Abeele and Leroy [49], [53], Van den Abeele [54], Van den Abeele and Leroy [56], and Van den Abeele et al. [62] extended the method as described in (44) but now with $I_{n} \equiv 1$ to form bounded beams, hence involving not only inhomogeneous waves having an inhomogeneity vector in one sense but also in the opposite sense. They studied in great detail the effects of frequency and beam width on the reflected beam profile. A later study was performed by Vanaverbeke et al. [86], following experimental research of Devolder et al. [99], on the possibility to measure the characteristics of a coating by means of phase-measuring (acousto-optic) techniques. One of their findings was that the results coming from the Fourier model correspond to the ones coming from the inhomogeneous wave model when studying the reflection of bounded beams on coated materials.

Nevertheless, the formation of bounded beams by means of inhomogeneous waves bares a drawback in that only an approximation can be found numerically in a relatively small area around the center of the beam. At large distances there are always exponentially growing amplitude tails. The presence of these tails can severely interfere with the areas of interest after reflection. Therefore, some numerical techniques have been developed [90], [91], [105] lately in order to shift those tails a little bit more outward. Furthermore, because the Prony technique only works for one variable, it is impossible to build 3-D bounded beams by means of inhomogeneous waves. Lately this shortcoming has been voided [95] by introducing a technique that is capable indeed of building 3-D bounded beams. A result of a 3-D Gaussian beam profile formed by means of a summation of inhomogeneous waves is shown in Fig. 5. Just as in the 2-D case, there appear exponentially growing tails at some distance away from the center of the profile.

Very recently, mainly because of the fact that bounded inhomogeneous waves have been experimentally generated (see further below), some papers have appeared dealing

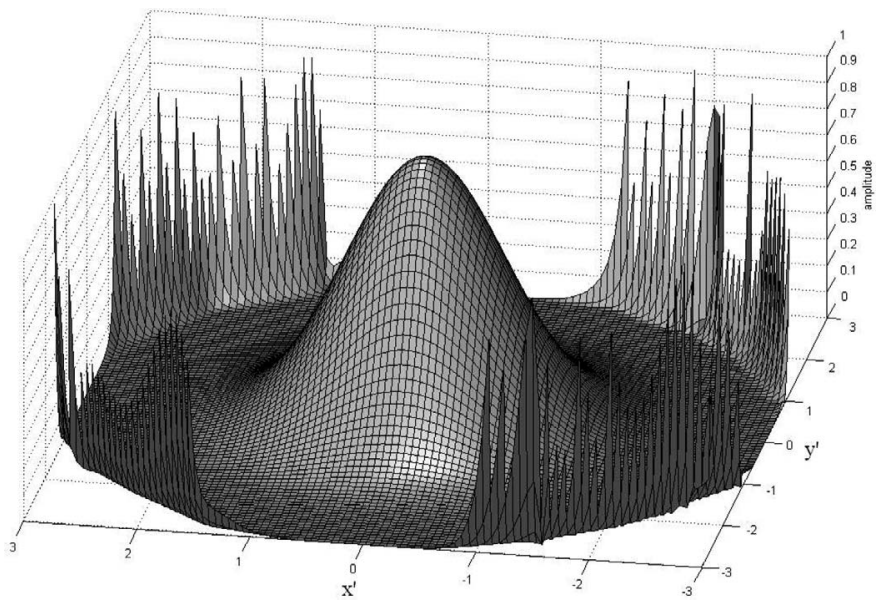

Fig. 5. The profile of a Gaussian beam in 3-D, built by means of a superposition of inhomogeneous waves.

with the description of the behavior of bounded inhomogeneous waves (i.e., an exponential profile but chopped in space) in reflection/transmission at plane interfaces between a liquid and a solid. The first description consists of the formation of bounded inhomogeneous waves by means of a Fourier decomposition into homogeneous plane waves, Vanaverbeke et al. [82]. Still, because it is interesting not just to simulate what happens to bounded inhomogeneous waves when they interact with materials, but also to understand what the physical and theoretical connection is between bounded inhomogeneous waves and infinite inhomogeneous waves, it was shown recently that a bounded beam is built up by means of infinite inhomogeneous waves and that the connection is made through the Laplace transform [92], [93]. Furthermore, it is shown that this description shows why bounded inhomogeneous waves behave like infinite inhomogeneous waves. The reason is that the infinite inhomogeneous wave having the same inhomogeneity as the bounded inhomogeneous wave determines what happens to the bulk of the bounded beam, but other inhomogeneities are only important to form the edges of the bounded beam.

\section{The Heritage From Electromagnetic Wave Theory}

Inhomogeneous waves were developed much earlier in electromagnetic wave theory than in ultrasonics theory. However, as is often the case in different branches of science, the connection between the two fields did not grow automatically. The first who connected inhomogeneous waves theory in ultrasonics with the one in electromagnetism was Hayes. In a paper which appeared soon after Atalar [7] and Claeys and Leroy [11], Hayes [9] published a theory dealing with inhomogeneous (surface) waves that was valid for both electromagnetic and ultrasonic waves. A few years later Hayes [16] published another paper in which he introduced the bivector formalism (see also [57]). The bivector formalism originates from Gibbs [100] and Synge [101], and Hayes revealed its importance in the description of ultrasonic and electromagnetic inhomogeneous 
waves. The bivector formalism of Hayes has formed the theoretical structure of inhomogeneous waves that is still used today.

\section{The Further Development of the Theory fOR Viscoelastic Isotropic Media}

Around 1984 the founders and the refounders of the theory of ultrasonic inhomogeneous waves had considered the existence of such waves: they had shown peculiarities of the reflection coefficient, they had considered the principle that later would be called the generalized law of Snell-Descartes (42), (43), and they had shown through the Schoch displacement that there was a strong connection between inhomogeneous waves and leaky surface waves. Nevertheless, a lot of work still needed to be done before the theory could reach its nowadays proportions.

Hayes [16] had already used the formalism of bivectors in the description of bulk inhomogeneous waves. Poirée [15] showed that this formalism also could be used to describe surface waves. Later Poirée's findings were extended by Poirée and Luppé [48]. Meanwhile Hosten and Deschamps [14] had applied the concept of complex angles (37) in the scattering of inhomogeneous waves. The latter two authors combined their efforts with the bivector formalism as used by Hayes [16] and Poirée [15] and extended the notion of damped homogeneous plane waves (reference 3 in [19]) and surface waves to inhomogeneous bulk waves. For the first time ever Hosten and Deschamps [14] published the dispersion relation in the bivector formalism [as is currently always used, see (29), (30)] for viscoelastic isotropic media; and, for the first time, they wrote about the generalized law of Snell-Descartes. The former was already calculated by Cooper and Reiss [2] and Cooper [3], the latter had already been obtained by Cooper and Reiss [2], Cooper [3], and Frisk [6], but here it was for the first time explicitly written within the bivector formalism. Hosten and Deschamps [14] showed within the bivector formalism the fact that inhomogeneous waves are generated naturally on an ideal liquid/absorbing solid and, therefore, complemented what had been tackled before by others [7], [11], [12].

The conservation of energy, which had been tackled before [26] for scattering of inhomogeneous waves on interfaces between ideal elastic media, was established by Deschamps [35] for viscoelastic materials. Hence, after 1990 there were no doubts possible anymore considering energy conservation when inhomogeneous waves get reflected/transmitted. A further study also can be found in Chevée and Deschamps [47] and Chevée [60]. Because the phase velocity of inhomogeneous waves is frequency dependent, it is also important to study the velocity of energy transport in such waves. This has been done by Poirée [46]. Because inhomogeneous waves were so astonishingly promising, Deschamps and Chevée [40] extended an approach (the expansion into a Debeye series) for describing the interaction of homogeneous plane waves with a solid layer immersed in a viscous liquid, to inhomogeneous waves. They found energy conservation laws. Nev- ertheless, they also found some peculiarities of inhomogeneous waves that were not so attractive for intuitive interpretation, such as propagation directions within the layer exceeding $90^{\circ}$, and they also found some convergence problems in their approach. Their paper in fact indicates that inhomogeneous waves can be considered as an excellent tool, but not the ultimate tool, for describing the interaction of sound with materials.

A treatise on the reflection/transmission of inhomogeneous waves (including shear horizontal waves) between two isotropic viscoelastic solids can be found in Caviglia and Morro [72]. The latter paper differs slightly from the other papers in the framework of inhomogeneous waves because the Stroh formalism is used here.

Lately [89] it also has been shown that inhomogeneous waves must be able to stimulate a surface wave on a liquid/solid interface that radiates both into the liquid and into the solid and, therefore, differs from a leaky Rayleigh wave.

\section{The Interaction of Ultrasonic InHOMOGENEOUS WaVES With ANisotropic MATERIALS}

Contrary to, for example, the book of Fedorov [102], it could be found already in the book of Musgrave [5] that complex wave vectors are mathematically possible in crystals. However, it was only much later that one has started to study this phenomenon deeply. The most general approach for describing the interaction of sound with an interface between generally anisotropic materials before 1986 is found in [20]. It is so general not only because of the possibility to calculate results for general anisotropy but also because the numerical approach is valid for all angles of incidence, but most earlier papers only presented results below the bulk critical angles. Furthermore, it has been shown by Lanceleur et al. [55] that Rokhlin et al. [20] must have implicitly implemented inhomogeneous waves because of correspondence with the later results of Lanceleur et al. [55]. One of the key factors is, of course, that Rokhlin et al. [20] determine the wave vector direction (up/down) by considerations of the energy flow when they obtain the correct choice of the sign, even in the case of generated inhomogeneous waves. Nevertheless, in Rokhlin et al. [20] there are no explicate studies described that reveal phenomena that are due to incident or scattered inhomogeneous waves. Therefore, the work of others [7], [11], [12], [14], where it was shown that damped waves produce inhomogeneous waves in a reflection/transmission process, motivated Hosten et al. [22] to describe transmitted waves in a viscous anisotropic material (e.g., a fiberreinforced composite) in the framework of inhomogeneous wave theory. The description of bulk inhomogeneous waves in anisotropic media had already happened by Hayes [16], but not in the context of reflection/transmission and certainly not their influence on the reflection/transmission coefficient. Hosten et al. [22] showed that the inhomogeneous wave theory produces results in agreement with ex- 
periments for propagation (through stiffness coefficients) and damping (through damping coefficients). This work was later further extended by Deschamps and Hosten [42] to a system in which the reflection/transmission coefficients are expanded in a Debeye series. Now because the vast majority of composites are layered, it was appealing to Hosten [41] to try numerical techniques that already existed for describing the interaction of homogeneous plane waves with layered (composite) materials (e.g., the Thomson-Haskell method) in the field of inhomogeneous plane waves. However, because the classical ThomsonHaskell method produces numerical instabilities for high values of frequency times thickness, Hosten [41] modified the method to get more stability and tested it in the case of damped materials, in which the then well established theory of inhomogeneous waves demanded the presence of bulk inhomogeneous waves inside the layered medium. Again he found excellent agreement with experiments. The rapid developments in inhomogeneous wave theory together with some important attempts to include such waves in reflection/transmission phenomena in anisotropic media (along crystal axes [22]) inspired Lanceleur et al. [55] to study the presence and the consequences of inhomogeneous waves due to reflection/transmission phenomena in general anisotropic media (therefore, also valid along the acoustical axes). Hence the paper of Lanceleur et al. [55] complements the paper of Rokhlin et al. [20]. For researchers in the field it, therefore, is necessary to study those papers together.

In addition, a thorough study on the differences of the Stroh formalism and the Christoffel equation formalism for inhomogeneous waves in anisotropic elastic materials can be found in Shuvalov [78].

Furthermore, it is well-known that, for homogeneous plane waves, the energy ray is directed along the direction of the wave vector, when this wave vector is directed perpendicular to the slowness surface in that direction. It is not always possible to have this situation, especially in anisotropic materials. Deschamps and Poncelet [85] showed that this is possible indeed if one deals with inhomogeneous waves because the inhomogeneity component becomes a steering factor. Hence, it is always possible to find such conditions for inhomogeneous waves. They also show that there are always four solutions in each direction.

Further findings of the interaction of inhomogeneous waves with anisotropic materials can be found in Deschamps and Assouline [76], Rogé [80], and Boulanger and Hayes [81]. In addition, the propagation of inhomogeneous waves in piezoelectric media has been considered by the first author of the current paper [108].

\section{Inhomogeneous Waves in Thermoviscous LIQUIDS}

In viscoelastic materials, there are only vibrational modes, i.e., (quasi) longitudinal and (quasi) shear modes. In thermoviscous liquids, however, there is also an en-

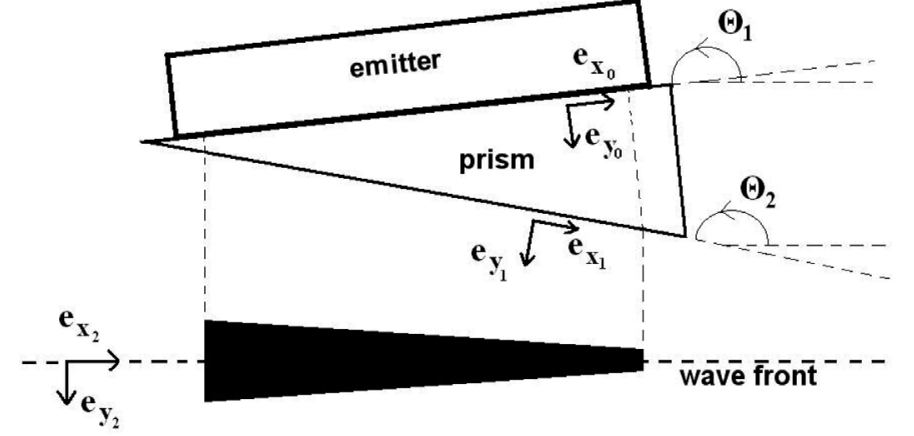

Fig. 6. The generation of an inhomogeneous wave by means of a damping prism. The black triangle represents the wave front, with the thickness being relative to the amplitude.

tropy (or thermal) mode. This mode, which formerly was only described as a pure homogeneous plane wave, was described in the framework of the inhomogeneous wave theory by Poirée [17], [23] for the case of a perfect thermoviscous gas by means of a first order perturbation of Navier-Stokes equations. Deschamps and Cheng [30] extended this model to the general case of thermoviscous liquids. The latter authors also showed that surface modes are possible that couple entropy modes with vibrational modes. The case of termoviscous solids can be found in Deschamps and Changlin [34].

\section{The Experimental Generation of Ultrasonic INHOMOGENEOUS WAVES}

Up until 1988, inhomogeneous waves had been important mostly from a theoretical point of view, and there were only indirect connections with experiments such as the Schoch effect or damping within materials. Most researchers in the field believed that bulk inhomogeneous waves could become an important tool for nondestructive evaluation (NDE); however, such waves had never been generated and studied before. Deschamps and Hosten [29] were the very first to build a tool for exciting bulk inhomogeneous waves, and they verified that experimentally excited bulk inhomogeneous waves had propagation properties in complete agreement with theory. From that moment on, bulk inhomogeneous waves became established physical entities. The excitation equipment consisted of a relatively wide transducer, and the inhomogeneous wave was generated by transmitting the generated sound through a damping prism-like material.

The principle is shown in Fig. 6. A homogeneous plane sound wave impinges the prism. Its characteristics are given by $\mathbf{k}_{0}=k_{1,0} \mathbf{e}_{x_{0}}$. Along the interface $y=x \tan \theta_{1}$ Snell's law must be fulfilled, when within the damping prism a damped homogeneous plane wave is generated. This wave is in its turn transmitted along the interface $y=x \tan \theta_{2}$ when also along this second interface it must fulfill Snell's law. Now by demanding that the transmitted wave (in the lossless lower media) must be conform 


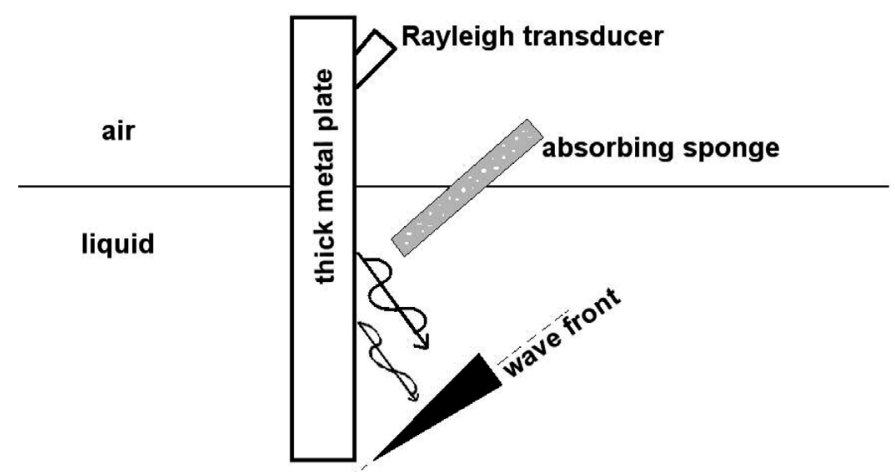

Fig. 7. A schematic of the generation of inhomogeneous waves by means of leaky Rayleigh waves. The black triangle denotes the wave front with thickness relative to the amplitude.

with $\mathbf{k}_{2}=\sqrt{\beta^{2}+(\omega / v)^{2}} \mathbf{e}_{x_{2}}-i \beta \mathbf{e}_{y_{2}}$, i.e., an inhomogeneous wave with given inhomogeneity vector $\boldsymbol{\beta}$, then it can be shown that implementation of Snell's law together with the dispersion relation inside the damping prism results in:

$$
\theta_{1}=\arctan \left(-p \beta \frac{v}{\sqrt{\beta^{2} v^{2}+\omega^{2}} v_{0}}\right)
$$

and

$$
\theta_{2}=-\arctan \left(-\omega \beta \frac{v}{\sqrt{\beta^{2} v^{2}+\omega^{2}} \alpha_{0} v_{0}}\right),
$$

with $p$ given by (51) (see next page) for $\alpha_{0}$ being the intrinsic damping and $v_{0}$ the sound velocity inside the prism.

The reason why both angles $\theta_{1}$ and $\theta_{2}$ must be tuned is the fact that not only the amplitude variation must be correct but also the wave front and its direction of propagation. It is, for example, seen from (49)-(51) that, if one demands $\beta=0$, that both angles are equal to $\pm \pi$. It also follows from (49)-(51) that, if one demands $\beta=\alpha_{0}$, that $\theta_{1}=\frac{\pi}{2}$ and that $\theta_{2}=\arctan \left(-\frac{v}{v_{0}}\right)$ which would be $-\frac{\pi}{4}$ in the case of $v=v_{0}$.

This method, using a damping prism, was also used in [75] in order to experimentally verify the theory of the diffraction of inhomogeneous waves on periodically rough surfaces (see further below).

A second method to experimentally generate bulk inhomogeneous waves has been presented by Huang et al. [58] and basically works as follows. Consider a thick metal plate which is half immersed in a liquid (Fig. 7). The other half is dry. When a Rayleigh transducer generates a pulsed Rayleigh wave on the dry side in the direction of the wet side, the generated Rayleigh wave will propagate toward the liquid. When it reaches the liquid, it becomes leaky. This leakiness causes sound to be transmitted at the Rayleigh angle into the liquid and also causes damping of the remaining Rayleigh wave in the solid plate. Hence, the amount that is leaked into the liquid diminishes along the propagation direction of the Rayleigh wave. This causes the radiated sound to be inhomogeneous, i.e., amplitude decay perpendicular to the propagation direction of the radiated sound. Furthermore, the leakage of sound into the liquid is frequency dependent, when the inhomogeneity in the generated bulk waves also is frequency dependent. An inhomogeneity then can be "chosen" for further research by filtering out all frequencies that do not produce this particular inhomogeneity. The drawback of the latter method is clearly the fact that the frequency and the inhomogeneity cannot be chosen independent from each other. The drawback of the former method is the fact that a different prism (i.e., different angles $\theta_{1}$ and $\theta_{2}$ ) is needed whenever a different inhomogeneity is required.

\section{The Diffraction of Ultrasonic INHOMOGENEOUS WAVES}

Since the 1980's, it was known that periodically corrugated surfaces possessed the ability to transform incident bulk waves into Scholte-Stoneley waves. That is because, under the right circumstances, one of the diffracted modes propagates along the interface under advantageous conditions. Therefore, such surfaces became appealing for nondestructive testing applications as for most materials it is otherwise not possible to stimulate Scholte-Stoneley waves on water-solid interfaces by means of sound incident from one of the two media. However, in nondestructive testing, it also is inviting to use Rayleigh waves. The problem arising from studies of the diffraction of pure plane waves (or wide bounded beams) on corrugated surfaces was that the leaky Rayleigh wave did not seem to get excited appropriately by this method. Hence, the combination of the inhomogeneous wave theory and the diffraction of plane waves was unavoidable. The most important difference between the diffraction of pure plane waves and the diffraction of inhomogeneous waves is that the grating equation must be generalized in a similar way as the law of Snell-Descartes had been generalized before.

The classical grating equation is given by:

$$
\sin \theta^{n}=\sin \theta^{i n c}+n \frac{\lambda}{\Lambda} .
$$

Unless one is attracted to working with complex angles, it is easier to transform this equation into:

$$
k_{x}^{n}=k_{x}^{i n c}+n \frac{2 \pi}{\Lambda} .
$$

The generalization, done by Briers and Leroy [50], [51], and Briers [64] consists of applying equation (53) not just for real wave vectors but also for complex wave vectors. Hence, it is seen from (53) that the diffraction phenomenon adds or subtracts a real value and not an imaginary value. In other words, the diffraction phenomenon does not alter $k_{2, x}$ but only $k_{1, x}$. The corresponding z-component of each of the diffracted waves then is found from the dispersion relation (15)-(16). Now because leaky Rayleigh waves and leaky Lamb waves have a complex wave vector component along the interface, it is seen from (53) that they 


$$
p=\frac{-\omega \alpha_{0} v \pm \sqrt{\beta^{2}\left(-v_{0}^{2} \beta^{2} v^{2}+\omega^{2}\left(v^{2}-v_{0}^{2}\right)\right)+\alpha_{0}^{2} v_{0}^{2}\left(\beta^{2} v^{2}+\omega^{2}\right)}}{v\left(\alpha_{0}^{2}-\beta^{2}\right)}
$$

cannot be stimulated by means of incident homogeneous plane waves and can be stimulated only by means of incident inhomogeneous waves. The results also can be found in later papers of Van Den Abeele et al. [62], [65]. The theory also was verified experimentally by Huang et al. [58] and Briers [75]. An extension of the model from homogeneous solids to porous media also can be found in Briers and Leroy [77]. Recently the theory of the diffraction of inhomogeneous waves clarified the backward beam displacement phenomenon on periodically rough surfaces as experimentally found by Breazeale and Torbett [87], [103] and connected this phenomenon to a leaky Scholte-Stoneley wave [88], [94]. Some theoretical predictions, based on the diffraction of inhomogeneous waves, have recently been verified experimentally [104]. Lately the theory of the diffraction of inhomogeneous waves on periodically corrugated surfaces in 1-D has been extended [84] to surfaces having a corrugation in 2-D. This theory shows that Scholte-Stoneley waves can be steered in the in-plane directions, depending on the frequency and the corrugation periodicities, but leaky Rayleigh waves cannot.

\section{Inhomogeneous Waves Having Complex FREQUENCY}

Inhomogeneous waves having complex frequency are generally called complex inhomogeneous harmonic plane waves. They also are called inhomogeneous transient waves. The imaginary part of the frequency corresponds to a source that has exponentially decaying (or growing) amplitude as a function of time. The main reason for studying such waves is the simple fact that perfect harmonic waves do not exist in nature. Every wave has started some time and will end to exist at some time. Especially in the field of geophysics, in which relatively short earthquakes generate transient surface waves or transient Lamb waves, complex (inhomogeneous) harmonic plane waves are becoming very important.

Consequences of complex frequency in the early stages of the theoretical development can be found in Borejko [25], Poirée [44], Deschamps [61], and Scott [63]. The real development of this theory started with Deschamps et al. [66].

For real frequencies, it is known that $k_{1}=\frac{\omega}{v}$, this is generalized for complex frequencies by working with the slowness vector $\mathbf{S}$, resulting in:

$$
\mathbf{k}=\omega \mathbf{S}
$$

when

$$
\begin{aligned}
& \mathbf{k}_{1}=\omega_{1} \mathbf{S}_{1}-\omega_{2} \mathbf{S}_{2}, \\
& \mathbf{k}_{2}=\omega_{2} \mathbf{S}_{1}+\omega_{1} \mathbf{S}_{2} .
\end{aligned}
$$

It is important to note here that the direction of the real wave vector and that of the real slowness vector are, in general, different form each other. It is also important to note that the components of $\mathbf{k}$ along a given Cartesian axis only depend on the components of $\mathbf{S}$ along that same axis. This means that relation (42) of the generalized SnellDescartes law also can be expressed in terms of $\mathbf{S}$ without any inconsistency.

Hence, for the case of complex frequencies, the dispersion relation (15) or (16) becomes for $m=d$ or $m=s$ :

$$
\begin{aligned}
\left(\omega_{1}^{2}-\omega_{2}^{2}\right) & \left(S_{1}^{2}-S_{2}^{2}\right)-4 \omega_{1} \omega_{2} \mathbf{S}_{1} \cdot \mathbf{S}_{2} \\
& =\frac{1}{v_{m}^{2}}\left(\omega_{1}^{2}-\omega_{2}^{2}\right)+2 \frac{\omega_{2}}{v_{m}} \alpha_{0, m}-\alpha_{0, m}^{2},
\end{aligned}
$$

and

$$
\left.\omega_{1} \omega_{2}\left(S_{1}^{2}-S_{2}^{2}\right)+\left(\omega_{1}^{2}-\omega_{2}^{2}\right) \mathbf{S}_{1} \cdot \mathbf{S}_{2}=\frac{\omega_{1} \omega_{2}}{v_{m}^{2}}-\frac{\omega_{1}}{v_{m}} \alpha_{0, m} .57\right)
$$

Relations (56) and (57) show that the complex frequency affects the spatial features of the sound wave.

Because it can be derived from (1) that the imaginary part will result in an exponentially decaying amplitude as a function of time if $\omega_{2}<0$ and exponentially growing if $\omega_{2}>0$, the particle displacement will not be an ellipse anymore as in the case of inhomogeneous waves having a real frequency, but it will be a collapsing elliptic spiral if $\omega_{2}<0$ and an expanding elliptic spiral if $\omega_{2}>0$.

An important question that might be posed in the case of transient inhomogeneous waves is, how fast do they travel? For inhomogeneous waves having real frequency, it is clear that they travel at the phase velocity. For transient waves, however, as a function of time you also get amplitude growth that is spreading in space. Therefore, it is necessary to distinguish between phase velocity:

$$
v_{p h}=\frac{\omega_{1}}{\mathbf{k}_{1} \cdot \mathbf{k}_{1}} \mathbf{k}_{1}
$$

and amplitude velocity

$$
v_{a m p}=\frac{\omega_{2}}{\mathbf{k}_{2} \cdot \mathbf{k}_{2}} \mathbf{k}_{2} .
$$

Furthermore, it can be found in Poncelet [70] that the velocity of energy propagation is given by:

$$
\mathbf{v}_{E}=\frac{\mathbf{S}_{1}}{\mathbf{S}_{1} \cdot \mathbf{S}_{p h}}
$$

with phase slowness vector $\mathbf{S}_{p h}$ given by:

$$
\mathbf{S}_{p h}=\frac{\mathbf{k}_{1}}{\omega_{1}}
$$


This shows that the energy propagates in the direction of $\mathbf{S}_{1}$.

Eq. (60) is the main reason why one who is dealing with transient waves prefers to work with the complex slowness vector $\mathbf{S}$ instead of the complex wave vector $\mathbf{k}$. Nevertheless, it always is possible to switch between slowness space and wave vector space by applying (55).

A thorough investigation of the energy flux for damped inhomogeneous complex plane waves in viscoelastic fluids can be found in Boulanger [73].

In the mid 1990's, one became interested in applying complex inhomogeneous harmonic waves for nondestructive testing. In 1996, Poncelet and Deschamps [69] started to study the generation, by means of such waves, of leaky Lamb waves in isotropic homogeneous plates. It is wellknown that the solutions (i.e., the possible wave vectors in the plane of the plate) of the Lamb wave characteristic equation (i.e., the dispersion equation for Lamb waves) for a fluid-loaded plate, are complex. This actually means that such waves can be generated by means of inhomogeneous waves and more generally by means of complex harmonic inhomogeneous waves. In their paper, Poncelet and Deschamps [69] show that the dispersion equation for Lamb waves can be expressed in the two independent unknown variables $\omega$ and $S_{x}$ (x-axis along the plane of the plate). This means that, for each Lamb mode $A_{n}$ and $S_{n}$, there is an infinite number of dispersion curves possible. For that reason a detailed study for the case of leaky inhomogeneous Lamb modes (i.e., complex slowness, real frequency) on the one hand and transient real Lamb modes (real slowness, complex frequency) on the other hand is performed. It is shown that the dispersion curves are different for both situations. Furthermore, it is shown that there is a huge difference for the $A_{0}$ and $S_{0}$ mode for complex frequencies if compared with real frequencies. The difference is that both modes do not converge to the Rayleigh wave velocity for thick plates, but stop to exist at a certain threshold real frequency times plate thickness. Also below this threshold there sometimes are two possible imaginary parts of the frequency for the same mode at the same real frequency times thickness. Furthermore, it is shown that, contrary to all other modes, the $A_{0}$ mode can be stimulated only with increasing amplitude as a function of time. A very important conclusion of complex harmonic inhomogeneous plane waves is perhaps the fact that they show that in time there is something similar (though not equivalent) as in space, which is that signals bounded in time are also capable of stimulating Lamb waves, like signals bounded in space (bounded beams) are capable of stimulating Lamb waves. An exotic study for the case of both complex wave vector and complex frequency reveals fantastic effects such as coupling of the branches of the $A_{0}$ with other modes. The findings of Poncelet and Deschamps [69] have later been experimentally verified in Deschamps and Poncelet [74].

Furthermore, in their paper, Poncelet and Deschamps [69] mention the important fact that the dispersion curves for complex frequencies correspond very well to the minima of the reflection coefficient calculated for an incident harmonic homogeneous plane wave, in contrast to the dispersion curves calculated for the solutions with complex slowness. This fact was later studied in full detail by Bernard et al. [79] for a plate immersed in a solid.

Lately, research has been done [96], [97] on the diffraction of transient waves on a periodically corrugated surface. It is found that transient plane waves are excellent tools to stimulate leaky Rayleigh waves on such surfaces, and it also is shown that such a surface can be used to filter any signal instantaneously into its complex frequency components. Lately, important effects of the complex frequency on the focal length of bounded beams have been discovered [107], [108] that can be very important for medical applications.

\section{Conclusions}

This paper has given an impression of what exists in the field of ultrasonic inhomogeneous waves, how each topic has started, and how it evolved. Perhaps the most important finding is that the theory has grown due to the strong interaction between the different researchers in this field. Therefore, it is not surprising that all given references form a network that cannot be divided into subgroups. This complicated network of collaborations in the past is also the reason why the references cannot be sorted by author. Hence, for historical reasons we have opted for predominantly chronological order, and we have described the evolution of this wonderful field in ultrasonics according to this time sequence. Whenever needed for clarity, we have selected subfields of inhomogeneous wave research and described its evolution as a separate paragraph, however, without hiding connections with other subfields in different paragraphs.

The theoretical development of ultrasonic inhomogeneous waves has more or less reached its final destination. Therefore, it is expected that the coming decade will be the one of practical applications in nondestructive testing and characterization of materials. Hence, there is still a huge world of opportunities for further research in the field of ultrasonic inhomogeneous waves and their applications. One of those studies has already been published [106], [108] and is dealing with the interaction of inhomogeneous waves with mud layers.

\section{REFERENCES}

[1] L. M. Brekhovskikh, Waves in Layered Media. New York: Academic, 1960.

[2] H. F. Cooper, Jr. and E. L. Reiss, "Reflection of plane viscoelastic waves from plane boundaries," J. Acoust. Soc. Amer., vol. 39, no. 6, pp. 1133-1138, 1966.

[3] H. F. Cooper, Jr, "Reflection and transmission of oblique plane waves at a plane interface between viscoelastic media," $J$. Acoust. Soc. Amer., vol. 42, no. 5, pp. 1064-1069, 1967.

[4] F. R. Spitzenogle and A. H. Quazi, "Representation and analysis of time-limited signals using a complex exponential algorithm," J. Acoust. Soc. Amer., vol. 47, no. 5, pp. 1150-1155, 1970. 
[5] M. J. P. Musgrave, Crystal Acoustics. San Francisco: HoldenDay, 1970.

[6] G. V. Frisk, "Inhomogeneous waves and the plane wave reflection coefficient," J. Acoust. Soc. Amer., vol. 66, no. 1, pp. 219-234, 1979

[7] A. Atalar, "Reflection coefficient for a lossy liquid-lossless isotropic solid interface," J. Acoust. Soc. Amer., vol. 65, no. 5, pp. 1101-1103, 1979.

[8] D. E. Weston and C. T. Tindle, "Reflection loss and mode attenuation in a Pekeris model," J. Acoust. Soc. Amer., vol. 66, no. 3, pp. 872-879, 1979.

[9] M. Hayes, "Energy flux for trains of inhomogeneous plane waves," Proc. R. Soc. Lond. A, vol. 370, pp. 417-429, 1980.

[10] D. E. Weston, "Oblique reflection of inhomogeneous acoustic waves," J. Acoust. Soc. Amer., vol. 69, no. 1, pp. 54-59, 1981.

[11] J. M. Claeys and O. Leroy, "How to choose a square root in calculating the reflection coefficient for a lossy liquid-lossless isotropic solid interface," J. Acoust. Soc. Amer., vol. 68, no. 6, pp. 1894-1896, 1980.

[12] A. Atalar, "On the reflection coefficient which exceeds unity," $J$. Acoust. Soc. Amer., vol. 70, no. 4, pp. 1182-1183, 1981

[13] J. M. Claeys and O. Leroy, "Reflection and transmission of bounded sound beams on half-spaces and through plates," $J$. Acoust. Soc. Amer., vol. 72, no. 2, pp. 585-590, 1982.

[14] B. Hosten and M. Deschamps, "Coefficients complexes de transmission ultrasonore à travers une lame a faces parallèles immergée," C. R. Acad. Sc. Paris, Serie II, vol. 299, pp. 519-522, 1984. (in French)

[15] B. Poirée, "Vitesse de propagation de l'énergie de l'onde plane évanescente acoustique," Rev. CETHEDEC, vol. 79, pp. 103112, 1984. (in French)

[16] M. Hayes, "Inhomogeneous plane waves," Arch. Ration. Mech. Anal., vol. 85, pp. 41-79, 1984.

[17] B. Poirée, "L'onde plane hétérogène dans un fluide thermovisqueux," Trait. Signal, vol. 2, pp. 223-226, 1985. (in French)

[18] J.-M. Claeys, "Theoretical models to describe reflection and diffraction of ultrasound from layered media," Ph.D. dissertation, Katholieke Universiteit Leuven Department of Sciences, Kortrijk, Belgium, 1985. (in Dutch)

[19] B. Hosten and M. Deschamps, "Génération d'ondes hétérogènes a l'interface liquide-solide viscoélastique. Approximation par des ondes inhomogènes," Acustica, vol. 59, pp. 193-198, 1986. (in French)

[20] S. I. Rokhlin, T. K. Bolland, and L. Adler, "Reflection and refraction of elastic waves on a plane interface between two generally anisotropic media," J. Acoust. Soc. Amer., vol. 79, no. 4, pp. 906-918, 1986

[21] O. Leroy, B. Poirée, L. Sebbag, and G. Quentin, "On the reflection coefficient of acoustic beams," Acustica, vol. 66, pp. 84-89, 1988 .

[22] B. Hosten, M. Deschamps, and B. R. Tittmann, "Inhomogeneous wave generation and propagation in lossy anisotropic solids. Application to the characterization of viscoelastic composite materials," J. Acoust. Soc. Amer., vol. 82, no. 5, pp. 1763-1770, 1987.

[23] B. Poirée, "Plane heterogeneous waves in a thermoviscous fluid," Acustica, vol. 64, no. 2, pp. 73-79, 1987.

[24] J. F. Allard and W. Lauriks, "Poles and zeros of the plane wave reflection coefficient for porous surfaces," Acustica, vol. 83, no. 6, pp. 1045-1052, 1997.

[25] P. Borejko, "Inhomogeneous plane waves in a constrained elastic body," Q. J. Mech. Appl., vol. 40, no. 1, pp. 71-87, 1987.

[26] O. Leroy, G. Quentin, and J. M. Claeys, "Energy conservation for inhomogeneous plane waves," J. Acoust. Soc. Amer., vol. 84 no. 1 , pp. $374-378,1988$

27] H. W. Jones, H. W. Kwan, and E. M. Yeatman, "A note on reflection and transmission of waves at a boundary between two viscoelastic media," J. Acoust. Soc. Amer., vol. 84, no. 3, pp. 1104-1106, 1988.

[28] L. Sebbag, "Les lois de la réflexion-réfraction des ondes planes évanescentes et les ondes d'interface," Master's thesis, University of Paris 7, 1987. (in French)

[29] M. Deschamps and B. Hosten, "Génération de l'onde hétérogène de volume dans un liquide non absorbant," Acustica, vol. 68, pp. 92-95, 1989. (in French)

[30] M. Deschamps and C. L. Cheng, "Ondes hétérogènes et réflexion-réfraction à l'interface liquide-non-absorbant/liquide- thermovisqueux," Acustica, vol. 68, pp. 96-103, 1989. (in French)

[31] B. Poirée, "Les ondes planes évanescentes dans les fluides parfaits et les solides élastiques," J. Acoustique, vol. 2, pp. 205-216, 1989. (in French)

[32] M. Deschamps and C. Changlin, "Réflexion-réfraction de l'onde plane hétérogène: Lois de Snell-Descartes et continuité de l'énergie," J. Acoustique, vol. 2, pp. 229-240, 1989. (in French)

[33] O. Leroy, K. Mampaert, and G. Quentin, "Inhomogeneous acoustic plane waves : generation, reflection and related phenomena," in Proc. Acousto-optics and Applications: 4th Spring School, May 23-27, 1989, Gdansk, pp. 153-174.

[34] M. Deschamps and C. Changlin, "Liquid-thermoviscoelastic solids interface," Ultrasonics, vol. 27, pp. 308-313, 1989.

[35] M. Deschamps, "Réflexion-réfraction de l'onde plane hétérogène: Répartition de l'énergie," J. Acoustique, vol. 3, pp. 251-261, 1990. (in French)

[36] B. Poirée and L. Sebbag, "Les lois de la réflexion-réfraction des ondes planes harmoniques évanescentes, I. Mise en équations," $J$. Acoustique, vol. 4, pp. 21-46, 1991. (in French)

[37] M. Deschamps and J. Roux, "Some considerations concerning evanescent surface waves," Ultrasonics, vol. 29, pp. 283-287, 1991.

[38] G. Quentin, A. Derem, and B. Poirée, "The formalism of evanescent plane waves and its importance in the study of the generalized Rayleigh wave," J. Acoustique, vol. 3, pp. 321-336, 1990.

[39] M. Deschamps, "L'onde plane hétérogène et ses applications en acoustique linéaire," J. Acoustique, vol. 4, pp. 269-305, 1991. (in French)

[40] M. Deschamps and P. Chevée, "Reflection and refraction of a heterogeneous plane wave by a solid layer," Wave Motion, vol. 15 , pp. $61-75,1992$

[41] B. Hosten, "Bulk heterogeneous plane waves propagation through viscoelastic plates and stratified media with large values of frequency domain," Ultrasonics, vol. 29, pp. 445-450, 1991.

[42] M. Deschamps and B. Hosten, "The effects of viscoelasticity on the reflection and transmission of ultrasonic waves by an orthotropic plate," J. Acoust. Soc. Amer., vol. 91, no. 4, pp. 2007-2015, 1992.

[43] J. M. Hovem and Å. Kristensen, "Reflection loss at a bottom with a fluid sediment layer over a hard solid half-space," $J$. Acoust. Soc. Amer., vol. 92, no. 1, pp. 335-339, 1992.

[44] B. Poirée, "Complex harmonic waves," in Physical Acoustics. O. Leroy and M. A. Breazeale, Eds. New York: Plenum, 1991, pp. 99-117.

[45] J. Roux, "Reflection and refraction of heterogeneous waves at plane interfaces," in Physical Acoustics: Fundamentals and Applications. O. Leroy and M. Breazeale, Eds. New York: Plenum, 1991, pp. 155-164

[46] B. Poirée, "Velocity of energy-transport in an acoustic complex plane wave," Acustica, vol. 74, no. 1, pp. 63-68, 1991.

[47] P. Chevée and M. Deschamps, "Interaction of plane heterogeneous waves within a damping layer-comparison between theory and experiment," Acustica, vol. 76, no. 5, pp. 224-230, 1992.

[48] B. Poirée and F. Luppé, "Evanescent plane waves and the Scholte-Stoneley interface wave," J. Acoustique, vol. 4, pp. 575$588,1991$.

[49] K. Van Den Abeele and O. Leroy, "Complex harmonic wave scattering as the framework for investigation of bounded beam reflection and transmission at plane interfaces and its importance in the study of vibrational modes," J. Acoust. Soc. Amer., vol. 93, no. 1, pp. 308-323, 1993.

[50] R. Briers and O. Leroy, "Reflection of inhomogeneous plane ultrasonic waves on periodically rough solid-vacuum interfaces," in Proc. SPIE, vol. 1844, 1992, Acousto-Optics and Applications, Gdansk-Jurata, Poland, pp. 196-205.

[51] R. Briers and O. Leroy, "Study of the behaviour of inhomogeneous harmonic waves in their diffraction from simple rough surfaces," J. Physique IV, vol. C1, no. 2, pp. 679-682, 1992.

[52] J. Duclos, O. Lenoir, J. M. Conoir, and J. L. Izbicki, "Description des ondes de Lamb en termes d'ondes evanescentes et de resonances," J. Physique IV, vol. C1, pp. 691-694, 1992. (in French)

[53] K. Van den Abeele and O. Leroy, "Bounded beam reflection and transmission effects in terms of complex harmonic waves," $J$. Physique IV, vol. C1, no. 2, pp. 675-678, 1992. 
[54] K. Van den Abeele, "Alternative fundamental theoretical descriptions for acousto-optic and acoustic investigation of pulsed and profiled ultrasound in view of nondestructive testing of layered structures," Master's thesis, Katholieke Universtiteit Leuven Campus Kortrijk, Belgium, 1992.

[55] P. Lanceleur, H. Ribeiro, and J.-F. De Belleval, "The use of inhomogeneous waves in the reflection-transmission problem at a plane interface between two anisotropic media," J. Acoust. Soc. Amer., vol. 93, no. 4, pp. 1882-1892, 1993.

[56] K. Van Den Abeele and O. Leroy, "On the influence and width of an ultrasonic bounded beam in the investigation of materials: Study in terms of heterogeneous plane waves," J. Acoust. Soc. Amer., vol. 93, no. 5, pp. 2688-2699, 1993.

[57] P. Boulanger and M. Hayes, Bivectors and Waves in Mechanics and Optics. London: Chapman and Hall, 1993.

[58] W. Huang, R. Briers, S. I. Rokhlin, and O. Leroy, "Experimental study of inhomogeneous wave reflection from a solid-air periodically rough boundary using leaky Rayleigh waves," J. Acoust. Soc. Amer., vol. 96, no. 1, pp. 363-369, 1994.

[59] G. Caviglia and A. Morro, "Wave-propagation in a dissipative stratified layer," Wave Motion, vol. 19, no. 1, pp. 51-66, 1994.

[60] P. Chevée, "Réflexion et transmission d'une faisceau borne ultrasonore sur une lame viscoélastique anisotrope immergée," Master's thesis, University of Bordeaux I, France, 1993. (in French)

[61] M. Deschamps, "Reflection and refraction of the evanescent plane wave on plane interfaces," J. Acoust. Soc. Amer., vol. 96, no. 5, pp. 2841-2848, 1994.

[62] K. Van Den Abeele, R. Briers, and O. Leroy, "Stimulated material vibration by inhomogeneous plane waves and its applications for bounded ultrasonic beams," in Proc. IEEE Ultrason. Symp., 1994, pp. 915-919.

[63] N. H. Scott, "Inhomogeneous plane waves in compressible viscous fluids," Wave Motion, vol. 22, pp. 335-347, 1995.

[64] R. Briers, "Contributions to the study of acoustic scattering and conversion phenomena in discontinuous structures by introducing a mode theory and by applying the inhomogeneous wave theory," Ph.D. dissertation, Katholieke Universiteit Leuven, Department of Sciences, Kortrijk, Belgium, 1995.

[65] K. E.-A Van Den Abeele, R. Briers, and O. Leroy, "Inhomogeneous plane-wave scattering and mode stimulation on periodic rough surfaces," J. Acoust. Soc. Amer., vol. 99, no. 5, pp. 28832897,1996

[66] M. Deschamps, B. Poiree, and O. Poncelet, "Energy velocity of complex harmonic plane waves in viscous fluids," Wave Motion, vol. 25, pp. 51-60, 1997.

[67] O. Leroy, "Nonspecular reflection-transmission phenomena of bounded beams described by inhomogeneous plane waves," in Acoustic Interaction with Submerged Elastic Structures, Part I. vol. 5, A. Guran, J. Ripoche, and F. Ziegler, Eds. World Scientific, Series on Stability, Vibration and Control of Systems Series B, pp. 129-163, 1996.

[68] O. Leroy, "Nonspecular reflection-transmission phenomena of bounded acoustic beams described by inhomogeneous plane waves," in Proc. Nordic Acoust. Meeting, June 12-14, 1996, pp. 491-494.

[69] O. Poncelet and M. Deschamps, "Lamb waves generated by complex harmonic inhomogeneous plane waves," J. Acoust. Soc. Amer., vol. 102, no. 1, pp. 292-300, 1997.

[70] O. Poncelet, "Ondes planes hétérogènes a fréquence complexe: Modes propres de plaques immergées," Ph.D. dissertation, University of Bordeaux 1, 1996. (in French)

[71] J. F. Allard and W. Lauriks, "Poles and zeros of the plane wave reflection coefficient for porous surfaces," Acustica, vol. 83, no. 6, pp. 1045-1052, 1997.

[72] G. Caviglia and A. Morro, "A new approach to reflectiontransmission between viscoelastic half-spaces," J. Acoust. Soc. Amer., vol. 106, no. 4, pp. 1666-1672, 1999.

[73] P. Boulanger, "Energy flux for damped inhomogeneous plane waves in viscoelastic fluids," Wave Motion, vol. 28, pp. 215-225, 1998.

[74] M. Deschamps and O. Poncelet, "Transient Lamb waves: Comparison between theory and experiment," J. Acoust. Soc. Amer., vol. 107, no. 6, pp. 3120-3129, 2000.

[75] R. Briers, O. Leroy, O. Poncelet, and M. Deschamps, "Experimental verification of the calculated diffraction field generated by inhomogeneous waves obliquely incident on a periodically rough liquid-solid boundary," J. Acoust. Soc. Amer., vol. 106, no. 2 , pp. $682-687,1999$.

[76] M. Deschamps and F. Assouline, "Attenuation along the Poynting vector direction of inhomogeneous plane waves in absorbing and anisotropic solids," Acustica-Acta Acustica, vol. 86, pp. 295-302, 2000.

[77] R. Briers and O. Leroy, "Surface mode generation at porous interfaces by permanent and transient heterogeneous plane waves," in Poromechanics. J.-F. Thimus, Y. Abousleiman, A.H.D. Cheng, O. Coussy, and E. Detournay, Eds. Rotterdam, The Netherlands: Balkema, pp. 179-183, 1998.

[78] A. L. Shuvalov, "On the theory of plane inhomogeneous waves in anisotropic elastic media," Wave Motion, vol. 34, pp. 401-429, 2001.

[79] A. Bernard, M. Deschamps, and M. J. S. Lowe, "Comparison between the dispersion curves calculated in complex frequency and the minima of the reflection coefficients for an embedded layer," J. Acoust. Soc. Amer., vol. 107, no. 2, pp. 793-800, 2000.

[80] B. Rogé, "Réflexion/transmission d'une onde plane inhomogene incidente sur une interface plane séparant deux milieux anisotropes," Master's thesis, Université de Technologie de Compiègne, France, 1999. (in French)

[81] P. Boulanger and M. Hayes, "Special inhomogeneous waves in cubic elastic materials," Zeitschrift fur angewandte mathematik und physik, vol. 51, no. 6, pp. 1031-1038, 2000.

[82] S. Vanaverbeke, F. Windels, and O. Leroy, "The reflection of bounded inhomogeneous waves on a liquid/solid interface," $J$. Acoust. Soc. Amer., vol. 113, no. 1, pp. 73-83, 2003.

[83] P. J. Wei and Z. M. Zhang, "Scattering of inhomogeneous wave by viscoelastic interface crack," Acta Mechanica, vol. 158, no. 3-4, pp. 215-225, 2002.

[84] N. F. Declercq, J. Degrieck, R. Briers, and O. Leroy, Diffraction of homogeneous and inhomogeneous plane waves on a doubly corrugated liquid/solid interface. Elsevier, to be published.

[85] M. Deschamps and O. Poncelet, "Inhomogeneous plane wave and the most energetic complex ray," Ultrasonics, vol. 40, pp. 293-296, 2002.

[86] S. Vanaverbeke, F. Windels, and O. Leroy, "The influence of coating thickness on the phase shift of bounded ultrasonic beams: Comparison between Fourier analysis and inhomogeneous plane wave theory," Acta Acustica United with Acustica, vol. 89, no. 4, pp. 640-646, 2003.

[87] N. F. Declercq, J. Degrieck, R. Briers, and O. Leroy, "Theoretical verification of the backward displacement of waves reflected from an interface having superimposed periodicity," Appl. Phys. Lett., vol. 82, no. 15, pp. 2533-2534, 2003.

[88] N. F. Declercq, J. Degrieck, R. Briers, and O. Leroy, "Theory of the backward beam displacement on periodically corrugated surfaces and its relation to leaky Scholte-Stoneley waves," $J$. Appl. Phys., vol. 96, no. 11, pp. 6869-6877, 2004.

[89] N. F. Declercq, J. Degrieck, and O. Leroy, "On the theoretical discovery of a new kind of leaky surface waves," in Paper P409 of the Proc. Tenth Int. Congr. Sound and Vibration, Jul. 7-10, 2003, Stockholm, Sweden.

[90] N. F. Declercq, J. Degrieck, and O. Leroy, "The principle of a chopped series equilibrium to determine the expansion coefficients in the inhomogeneous waves decomposition of a bounded beam," Acta Acustica United with Acoustica, vol. 89, pp. 10381040, 2003.

[91] N. F. Declercq, J. Degrieck, and O. Leroy, "Two improved methods to determine the coefficients in the inhomogeneous wave decomposition of bounded beams," in Peer reviewed Section of the Proc. 8th Western Pacific Acoustics Conf. (Wespac8), vol. paper WE41, Apr. 7-9, 2003, Melbourne, Australia.

[92] N. F. Declercq, J. Degrieck, and O. Leroy, "On the ability of bounded inhomogeneous waves to experimentally verify the behavior of infinite inhomogeneous plane waves," J. Acoust. Soc. Amer., vol. 113, no. 4, p. 2283, 2003.

[93] N. F. Declercq, J. Degrieck, and O. Leroy, "The Laplace transform to describe bounded inhomogeneous waves," J. Acoust. Soc. Amer., vol. 116, no. 1, pp. 51-60, 2004.

[94] N. F. Declercq, J. Degrieck, R. Briers, and O. Leroy, "A theoretical elucidation for the experimentally observed backward displacement of waves reflected from an interface having superimposed periodicity," J. Acoust. Soc. Amer., vol. 112, no. 5, p. $2414,2002$. 
[95] N. F. Declercq, J. Degrieck, and O. Leroy, "The inhomogeneous wave decomposition of 3D Gaussian-like bounded beams," Ultrasonics, vol. 42, pp. 273-276, 2004.

[96] N. F. Declercq, J. Degrieck, and O. Leroy, "Diffraction of transient harmonic plane waves and the stimulation of transient leaky Rayleigh waves," submitted for publication.

[97] N. F. Declercq, J. Degrieck, and O. Leroy, "The acoustic diffraction grating: A complex frequency filter device for electronic signals," submitted for publication.

[98] H. L. Bertoni and T. Tamir, "Unified theory of Rayleigh-angle phenomena for acoustic beams at liquid-solid interfaces," Appl. Phys., vol. 2, pp. 157-172, 1973.

[99] S. Devolder, M. Wevers, P. DeMeester, and O. Leroy, "Thin layer thickness measurements based on the acousto-optic technique," Appl. Phys. Lett., vol. 68, no. 12, pp. 1732-1734, 1996.

[100] J. W. Gibbs, "Elements of vector analysis," in Scientific Papers. vol. 90, no. 2, New York: Dover Publications, 1961, pp. $17-90$.

[101] J. L. Synge, "The Petrov classification of gravitational fields," in Comm. Dublin Inst. Adv. Studies. ser. A, no. 15, Dublin, 1966.

[102] F. I. Fedorov, Theory of Elastic Waves in Crystals. New York: Plenum, 1968.

[103] M. A. Breazeale and M. Torbett, "Backward displacement of waves reflected from an interface having superimposed periodicity," Appl. Phys. Lett., vol. 29, no. 8, pp. 456-458, 1976.

[104] A. Teklu, M. A. Breazeale, N. F. Declercq, R. D. Hasse, and M. S. McPherson, "Backward displacement of ultrasonic waves reflected from a periodically corrugated interface," Appl. Phys. Lett., 084904 pp. 1-4, 2005.

[105] N. F. Declercq, J. Degrieck, and O. Leroy, "A useful analytical description of the coefficients in an inhomogeneous wave decomposition of a symmetrical bounded beam," Ultrason., vol. 43, no. 4, pp. 279-282, 2005.

[106] N. F. Declercq, O. Leroy, J. Degrieck, and J. Vandeputte, "The interaction of inhomogeneous waves and Gaussian beams with mud in between a hard solid and an ideal liquid," Acta Acustica United with Acustica, vol. 90, pp. 819-829, 2004. Degrieck Leroy

[107] N. F. Declercq, J., O., "Focal length control of complex harmonic and complex pulsed ultrasonic bounded beams," J. Appl. Phys., vol. 97, no. 5, pp. 1-8, 2005.

[108] N. F. Declercq, "The interaction of complex harmonic elastic waves with periodically corrugated surfaces and with anisotropic viscoelastic or piezoelectric layered media," Ph.D. dissertation (Applied Sciences: Physics), Ghent University, Faculty of Engineering, 2005.

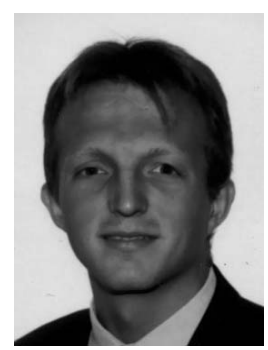

Nico F. Declercq was born in Kortrijk (Belgium) in 1975 and obtained a B.Sc. and M.Sc. degree in (Astro-)physics at the Catholic University of Leuven. He began working as a volunteer researcher in The Physical Acoustics and Acousto-Optics Research Group of O. Leroy at the Interdisciplinary Research Center and moved to the Ghent University in October 2001 where he started his Ph.D. research with financial support of 'The Flemish Institute for the Encouragement of the Scientific and Technological Research in Industry (I.W.T.)'. The public defense of his Ph.D. dissertation is expected to be held in April 2005. Meanwhile, he has published over 25 papers in journals, listed in the science citation index, and has made a few dozens of presentations at international congresses. He is also a regular referee for several journals. His current theoretical and experimental research is situated in Physical Acoustics (diffraction on corrugated interfaces, interaction of sound with isotropic and anisotropic piezoelectric and non-piezoelectric materials, interaction of sound with mud and sediment layers, inhomogeneous waves, etc.), Architectural Acoustics (diffraction phenomena on staircases, etc.), Underwater Acoustics (ultrasonic beams, etc.) and Acousto-Optics (Schlieren photography, nondestructive testing, etc.). He is a member of IEEE, Acoustical Society of America ASA, Russian Acoustical Society RAS, Belgian Acoustical Society ABAV, French Acoustical Society SFA, The British Institute of Non-Destructive Testing BINDT, The International Institute of Acoustics and Vibration IIAV and the Acoustical Society of India ASI, The Canadian Acoustical Association CAA-ACA, The American Physical Society APS and The European Physical Society EPS. He has won the Best Presentation Award at the 2nd FTW Ph.D. symposium (Ghent University 2001) and the Physical Acoustics Best Student Paper Award presented by the Physical Acoustics Technical Committee of the Acoustical Society of America at the First Pan-American/Iberian Meeting on Acoustics (Cancun, Mexico, 2002). He is the coordinator of a Nato grant in collaboration with The Institute of Radio Engineering and Electronics (Russian Academy of Sciences, Moscow, Russia) and The National Center for Physical Acoustics (Ole Miss, MS). Lately, he has been a visiting scientist at The Laboratory for Acousto-Optics and Solid State Acoustics (NCPA-Ole Miss, MS), Precision and Intelligence Laboratory (Tokyo Institute of Technology, Japan), The Department of Materials Engineering (University of Moratuwa, Sri Lanka), The Laboratory of Acoustic Microscopy (Institute of Biochemical Physics, Russian Academy of Sciences, Moscow, Russia) and The Institute of Radio Engineering and Electronics (Russian Academy of Sciences, Moscow, Russia) and The Department of Physics (M. V. Lomonosov Moscow State University, Moscow, Russia). He also assists in organizing the World Congress on Ultrasonics. Furthermore, his work on special acoustic effects in front of the Great Pyramid of Chichen Itza (Mexico) has been reported by Nature and numerous newspapers around the globe.

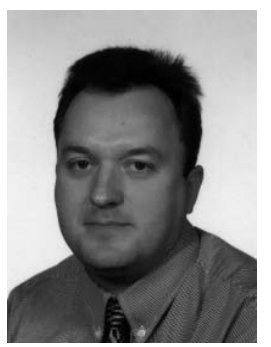

Rudy Briers was born in Geel (Belgium) on October 9th 1965, has an B.Sc. and M.Sc. in mathematics, is aggregated for teaching in higher education and has a Ph.D. in Sciences at the Catholic University of Leuven (KUL, Belgium). He has been assistant professor in the department of Astronomy and the department of Mathematics and Physics. After his Ph.D., he worked as a postdoctoral fellow and was a driving force at the Physical Acoustics and Acousto-Optics Research Group of Prof. O. Leroy, with financial support of the Flanders Institute for Scientific Research and Technology (IWT) and the Belgian National Fund for Scientific Research (FWO). His research is mainly situated in the field of Physical Acoustics (non-destructive testing, coatings, diffraction gratings, surface waves, etc.). He developed the Radiation Mode Theory in ultrasonics in collaboration with Prof. O. Leroy and Prof. G. Shkerdin (Russian Academy of Sciences). At present, he is a lecturer in Mathematics and Computer Sciences at the Katholieke Hogeschool Zuid-West Vlaanderen (KATHO, Belgium) and is a professor of Statistics in Medical Sciences at the Catholic University of Leuven. He is referee for international journals and member of several acoustical societies.

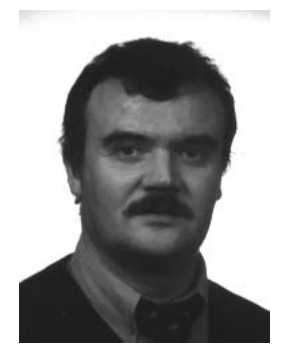

Joris Degrieck was born in Veurne (Belgium) on the 10th of February 1959 and obtained an M.Sc. in Mechanical Engineering and a Ph.D. in Applied Science (Mechanical Engineering) at the Ghent University. From 1986-1991 he has been assistant and lecturer at the Ghent University. From 1991-1996, he has been a research engineer and project leader at the Research Institute of the Belgian Metalworking Industries (WTCM-CRIF). From 1993 until present, he has been respectively part time professor, teaching "Mechanics of Fibre Reinforced Materials" and professor, teaching "Composite Materials", "Mechanics of Materials", and "Kinematics and Dynamics of Mechanisms" at the Ghent University. His main research activities are situated in mechanics of fiber reinforced composites under impact and fatigue loading and non destructive characterization of the elastic properties of composite laminates 
by means of ultrasound. He has won The Vreedenburgh Prize (TU Delft, the Netherlands, 1991) and The prize of the Belgian Royal Academy for Arts, Letters and Sciences - Class Sciences (1991).

$\mathrm{He}$ is a member of The Flemish Engineering Association KVIV, The Society for the Advancement of Material and Process Engineering, The Materials and Processes Society SAMPE, The Belgian Research Centre for Pipes and Equipment Becetel, The Belgian Society of Mechanical and Environmental Engineering BSMEE, member and council member of The European Society for Composite Materials ESCM and is an affiliate member of The Institute of Materials. At present, he is the Head of the Department of Mechanical Construction and Production (Soete Laboratory) at the Ghent University. He is referee for several journals and contractor for numerous companies.

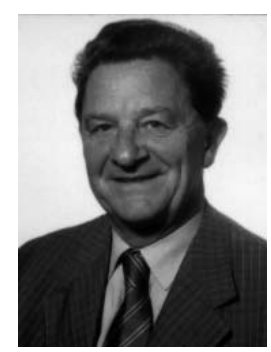

Oswald Leroy was born in Passendale (Belgium) in 1936 and received his Ph.D. in Mathematical Physics at the Ghent University. His main fields of interest are AcoustoOptics and Physical Acoustics. His work has resulted in many scientific advances in the famous so called Belgian School of Acousto Optics (Founded by R. Mertens after WWII). He worked as an assistant at the Ghent University from 1966 until 1972 and has been Professor at the Catholic University of Leuven (Katholieke Universiteit Leuven) since
1972, where he established and directed the Physical Acoustics and Acousto-Optics Research Group at the Interdisciplinary Research Center. He was a Guest Professor at Université de Paris 7 and Université de Bordeaux in France, University of Tennessee in the U.S. and Tokyo Institute of Technology in Japan. He has been a referee for many International Journals. He received Nato Grants in cooperation with Georgetown University, University of Tennessee and University of Houston.

He is a Fellow of The Acoustical Society of America, and a member of The Institute of Electric and Electronic Engineering, Société Française d'Acoustic and The Belgian Acoustical Society. He received an Honorary Doctorate from the University of Gdansk (Poland) in 1991. He has won the Médaille étrangère of the French Acoustical Society in 2001. He accorded his Emeritus Status in October 2001. 